\title{
Balance the Energy Consumption of Geographic Hashing Location Service in Wireless Sensor and Actor Networks
}

\author{
https://doi.org/10.3991/ijoe.v15i08.9419 \\ Zhaolin Wang, Zhezhuang Xu ${ }^{(凶)}$, Renxu Xie \\ Fuzhou University, Fuzhou, China \\ zzxuefzu.edu.cn \\ Haotian Yan \\ Hong Kong Polytechnic University, Hong Kong, China
}

\begin{abstract}
Location service is an efficient solution to handle actor mobility in wireless sensor and actor networks. Geographic hashing location service (GHLS) is a flat hashing-based protocol which has better energy efficiency than other location service protocols. Nevertheless, GHLS suffers from unbalanced energy consumption due to the fixed hashed region. In this paper, we propose a new protocol termed as GHLS-R2 to achieve load balance in two aspects: location server rotation and energy-aware geographic routing. Simulation results show that GHLS-R2 protocol can effectively balance the energy consumption, and hence prolong the network lifetime significantly.
\end{abstract}

Keywords - Location service, Energy Efficiency, Location server rotation, Geographic routing, Wireless sensor and actor networks

\section{$1 \quad$ Introduction}

In recent years, wireless sensor and actor networks (WSAN) [1] become popular in applications due to their low cost and easy deployment. The static sensor nodes can be used to monitor the event of interests [2], [3], while the mobile actors move in the network area to collect data from sensor nodes and then deal with the events. However, the location information of the mobile actors is always unavailable for the sensor nodes due to the actor mobility. Thus, how to send the event report to the mobile actor is a significant issue.

To solve this problem, a popular solution is location service [4-6]. In location service, the mobile actors should send update packets to a set of nodes called location servers. The update packets contain the actors' latest location. When the event is detected, the source node, whose location is close to the locale of the event, sends query packets to the location servers to obtain the actor's location. Then the event report can be delivered to the actor by using the geographic routing [7].

Some research works have proposed different algorithms to select location servers, such as XYLS [8], GLS [9] and GHLS [4]. XYLS is a proactive location service that disseminates locations in column direction such that a query which transmits in row 
direction can intersect it subsequently. GLS is a hierarchical hashing-based location service which disseminates each node's location to $O(\log N)$ nodes. Geographic hashing location service(GHLS) is a flat hashing-based protocol. In GHLS, it hashes the node's unique name into a location and the node whose location is closest to the hashed location is selected as location server. GHLS has been proved to have best energy efficiency than other location service protocols.

GHLS is originally designed for mobile ad-hoc networks, thus the location server for each mobile node is hashed into a fixed region. However, in WSAN, the sensor nodes are fixed to monitor the area. In this case, the location server is fixed along the whole network lifetime, thus the unbalanced energy consumption becomes a critical issue.

In this paper, we study the unbalanced energy consumption of GHLS in WSAN. Then we propose an improved protocol named GHLS- $\mathrm{R}^{2}$ to balance the energy consumption and prolong the network lifetime. The major contributions of this paper are given as follows.

- The numerical analysis is provided to study the unbalanced energy consumption of GHLS. The simulation results show that less than $2 \%$ energy is consumed when the first dead node appears. The reason is that the location server consumes much more energy than other nodes. The results motivate us to propose GHLS- $\mathrm{R}^{2}$ to balance the energy consumption.

- The location server rotation is proposed in GHLS- $\mathrm{R}^{2}$ to balance the overwhelming energy consumption around the location server. The rotation period is also studied to improve energy efficiency.

- GHLS-R ${ }^{2}$ adopts the energy-aware Greedy-Face-Greedy (GFG) routing which considers both geographic distance and residual energy in the routing decision, and thus balances the energy consumption in the data transmission process.

- Simulations are executed to prove that both location server rotation and energyaware GFG routing is helpful to balance the energy consumption, thus GHLS-R ${ }^{2}$ can improve the network lifetime significantly.

The rest of this paper is organized as follows. Section 2 provides a brief introduction of related works. Section 3 introduces the network model, GHLS protocol and the problem addressed in this paper. The details of GHLS-R ${ }^{2}$ protocol are given in Section 4. Section 5 shows the simulation results to prove the efficiency of GHLS- ${ }^{2}$.Section 6 makes the conclusion of this paper.

\section{$2 \quad$ Related Works}

In this section, a brief survey is provided to introduce the related works of location service algorithms. The main idea of location service is to use the location information, which can be obtained by GPS or localization technologies [10], for mobility management in the wireless sensor and actor networks. In location service, the actor has to periodically update its location to several sensor nodes which are defined as location servers. When a sensor node needs to transmit a packet to the actor, it has to 
query the recorded location of the actor from location servers, then the geographic routing can be used for data transmission to the actor.

According to the schemes of location server selection, location service algorithms can be further divided into quorum-based and home-based algorithms [11]. The rest of this section is organized in these two aspects.

XYLS (column-row location service) [8] is a typical quorum-based location service. In XYLS, the location update packet is forwarded to a vertical area, while the location query packet has to be transmitted to a horizontal area. The intersection of these two areas becomes a rendezvous area where the query is guaranteed to be satisfied. Many location service algorithms are developed based on XYLS, such as the works in [12], [13]. In MLS (Mobility strategy-based Location Service) [12], the mobility strategy of the actor is contained in the update packet, such that the location update period can be greatly reduced. TLS (Trail-based Location Service) [13] exploits the trail of the actor to improve the reliability and the energy efficiency of the quorum-based location service. On the other hand, some works change the shape of the quorum. The Local Update-based Routing Protocol (LURP) [14] is proposed to constrain the location update in a local area, and the area is built according to the actor mobility. The performance is further improved by ALURP [15] in which the actor can adjust its location update range adaptively. The authors in [16] adopt quorum-based schemes to real irregular fields with voids as well as non-rectangular shapes and propose an energy-balanced location service scheme.

The home-based location service uses a public hash function to select location servers [4], [5]. The ID of the mobile actor is the input to the hash function, and the output can be either the ID or the geographic location of the location server. The paper [17] compares the performance of the HLS (Hierarchical Location Service) and GLS (Grid Location Service) [5] coupled to the GPSR (Greedy Perimeter Stateless Routing) algorithm. It presents the efficiency and the scalability performance study of both GLS and HLS, and analyzes their applicable scenes respectively. The LBDD (Line-based data dissemination) [18] uses a virtual vertical line or strip area constructed in the central field of the network which can divide the whole network into two equal parts. The source transmits the data packet towards the virtual area. When the actor requests event reports, it also sends a query to this rendezvous area. Then it travels linearly until it encounters the node storing the packets. The paper [19] proposes the DRSS protocol (Directional Routing and Scheduling Scheme) where the source should forward the event packets to a predetermined rendezvous area. The event packets are stored in the rendezvous area until a mobile actor moves nearby to collect them.

GHLS [4] uses geographic hashing to map the location server, which is denoted as $S$, to a geographic location $H(S)$. Each node is assigned a single location server, by hashing the node's unique name into a location in the geographic area, and designating the node whose location is currently closest to the hashed location as the node's location server. GHLS has been proved to be a reasonable solution for location service due to its efficiency and simplicity. GHLS is originally proposed for mobile adhoc networks, where most nodes are mobile. However, in wireless sensor and actor networks, most sensor nodes are static and with limited energy, thus the energy bal- 
ance becomes an important problem. The details and problems of GHLS are introduced in Section 3.

Compared with related works, the GHLS- $\mathrm{R}^{2}$ protocol proposed in this paper distinguishes them in the following aspects:

- GHLS-R ${ }^{2}$ uses location server rotation to balance the overwhelming energy consumption near the location server.

- GHLS- $\mathrm{R}^{2}$ uses energy-aware Greedy-Face-Greedy (GFG) routing to balance the energy consumption in the data transmission process.

- This paper makes in-depth study about the parameters setting including the rotation period and the routing factor.

\section{$3 \quad$ Preliminaries}

This section firstly introduces the network model considered in this paper. Then we introduce the GHLS protocol, and study its problems that motivates the works of this paper.

\subsection{Network model}

In this paper, the wireless sensor and actor network is assumed to have the following properties:

- The network which contains $N$ sensor nodes and one actor. The sensor nodes are randomly deployed in a square area whose side length is defined as $L$. The sensor nodes monitor the network area and detect the interest event.

- The actor movement follows the random waypoint model [20]. In this model, the actor chooses a destination randomly in the network area. Then, the actor moves straight to this destination with speed $\mathrm{v}$. When the actor reaches the destination, a new destination will be chosen randomly and the actor moves to the new destination. The process repeats without pause time.

- Both sensor nodes and the actor can use GPS or localization algorithms [10], [21] to obtain their locations. Thus the geographic routing, such as GFG routing, can be used for data transmission.

- Sensor nodes are powered by batteries and have limited energy. Thus the load balance and energy efficiency are significant issues [22].

- The sensor node or actor broadcasts its data packet with limited transmission range $\left(R_{t}\right)$, and the network is ensured to be connected with $R_{t}$ [23], [24]

\subsection{Geographic hashing location service (GHLS)}

Geographic hashing location service (GHLS) is a flat hashing-based location service which uses hash function to build a home region [4] that contains location servers. The mobile actor has to periodically forward an update packet, which contains its 
latest location, to the location server. On the other hand, when an event of interest is detected, the source node firstly sends a location query packet to the location server to obtain the actor's location and then transmits the event report to the mobile actor. The details of GHLS are given as follows.

Selecting home region: Home region locates at the center of network area. The size of the home region is changed by a scaling factor $K$, where $K$ is the ratio of the home region area to the network area. When $K=0$, the home region is the geographic center point of the network area. When $K=1$, the home region is the whole network area.

Selecting location server: The location server is determined by a geographic hash function. The hash function can hash the actor's unique name into a location in home region. Then the sensor node, whose location is currently closest to the hashed location, is selected as location server. The unique name can be IP address or MAC address.

Location update: When the location server is determined, the actor sends update packet, which contains its latest location, to the location server in every update period.

Data transmission: The source uses the same hash function to obtain the location of location servers. The source firstly sends the query packet to the location server and obtains the latest location of the actor. Then the event report is forwarded to the actor via geographic routing. However, due to the mobility, the location saved in location server is not exactly the actor's location at this moment. Thus, the event report is forwarded to the last-hop node which is closest to the location saved in location server. Generally, the actor locates in the transmission range of the last-hop node.

Geographic routing: In GHLS, the packets can be delivered to the destination by using a geographic routing algorithm called Greedy-Face-Greedy (GFG) routing. The GFG routing runs in a greedy manner that forwards the packet to the next-hop node which is closest to the destination.

\subsection{Unbalanced energy consumption in GHLS}

GHLS has been proved to have better energy efficiency than other location service protocols when $K=0$, i.e. the location server is fixed at the center of the network area. Since it is originally proposed for location service in mobile ad-hoc networks, it does not consider the load balance in the network. However, the load balance is very important in WSAN which has numerous sensor nodes with limited energy. Therefore, we decide to execute simulations to study the energy consumption of GHLS in WSAN.

In the simulation, we use the network lifetime and the residual energy ratio as metrics to evaluate the load balance in GHLS. The network lifetime is defined as the time when the first node dies in the network, and the residual energy ratio is defined as the ratio of residual energy to initial energy when the first dead sensor node appears. To clarify the analysis, the energy consumption is unified that one unit of energy is equal to the energy consumption for transmitting a packet with fixed transmission range $R_{t}$.

The node density of the network is 0.01 node $/ \mathrm{m}^{2}$. The maximum transmission range $R_{t}$ is $60 \mathrm{~m}$. The velocity of the actuator node is $2 \mathrm{~m} / \mathrm{s}$. The update period is 30 
seconds that is dividing the maximum transmission range by the actuator velocity to ensure network connectivity. The initial energy of every node is 50 units. Moreover, the energy of the location server, denoted as $E_{L}$, is set as 50 and 5000 respectively. The reason for setting $E_{s}=5000$ is to study the unbalanced energy consumption around the location server. For sensor nodes expect the location server, their energies are set as 50. The simulation program is implemented in Matlab, and each experiment is carried out 50 times to obtain the statistical results.

Fig. 1 shows that the network lifetime is extremely low due to the heavy traffic load of location updates and queries on the location server. The results given in Fig. 2 show that more than $98.9 \%$ energy is left in the network when the first dead node appears. The major reason is that the hashed region is fixed in GHLS, which leads to the fixed location server in WSAN.

On the other hand, as shown in Fig.1, increasing the energy of the location server is helpful to improve the network lifetime. However, the energy consumption is still severely unbalanced. Fig. 2 shows that more than 95.6\% energy is left when the first dead node appears. It is because the sensor nodes around the location server still suffer from the heavy traffic load. This result indicates that simply considering the energy consumption of the location server is insufficient to solve the problem.

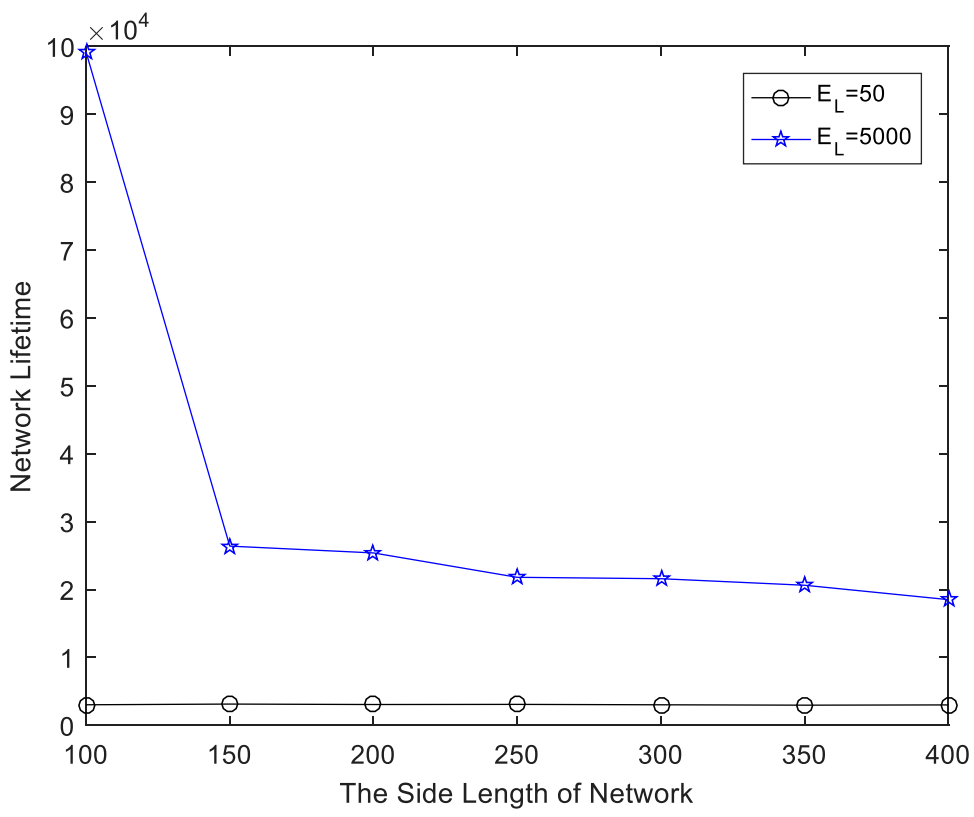

Fig. 1. The network lifetime with different network area

The simulation results given above prove that the energy consumption of GHLS is severely unbalanced in WSAN. It motivates us to propose an improved protocol based on GHLS to balance the energy consumption. The details are given in the next section. 


\section{GHLS-R ${ }^{2}$ Protocol}

In this paper, we propose a protocol named GHLS- $\mathrm{R}^{2}$ to balance the energy consumption of GHLS in WSAN.GHLS- ${ }^{2}$ balances the energy consumption in two aspects: At first, the location server has to rotate periodically in the network to balance the energy consumption. In addition, the energy-aware GFG routing is proposed to avoid selecting nodes with low energy during the routing process.

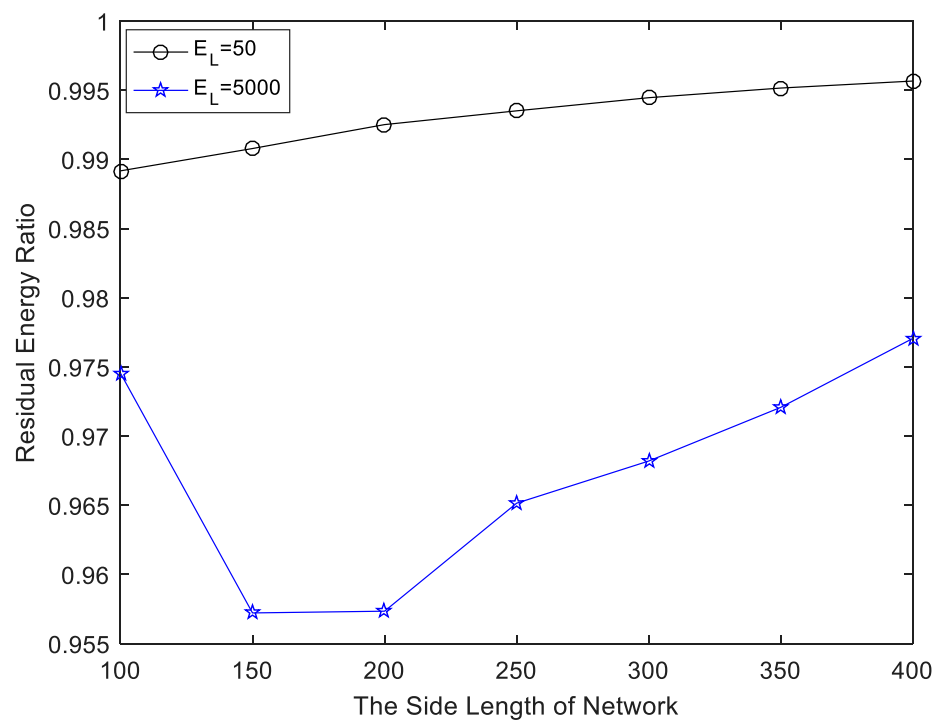

Fig. 2. The residual energy ratio with different network area

The framework of GHLS- ${ }^{2}$ is developed based on GHLS, thus the schemes of location update and data transmission in GHLS- $\mathrm{R}^{2}$ are the same as the schemes in GHLS. Please refer to Section 3.2 for details. In the rest of this section, we will introduce the details of location server rotation and energy-aware GFG routing in GHLS$\mathrm{R}^{2}$.

\subsection{Location server rotation}

The fixed location server is the most significant factor that generates unbalanced energy consumption in GHLS. Thus, in GHLS- $\mathrm{R}^{2}$, we propose that the location server has to rotate every period based on a random hash function,

$$
H=f(A, t)
$$

Where $H$ denotes the coordinates of the hashed location, $A$ is the ID of the mobile actor and $t$ denotes the current time slot. The location server is the sensor node which 
is closest to the hashed location. Every node should have the same random seed, such that the location update and location query packets can be forwarded to the same location server.

The rotation of location server is divided in periods, which is controlled by the rotation period $T_{s}$.

$$
T_{s}=s \times T_{u}
$$

Where $T_{u}$ is the location update period and $s$ is a factor to control the value of $T_{s}$. The impact of $T_{u}$ will be analyzed in Section 5.1. It is worthy to note that, the actor has only one location server during every period. Thus, when a new node has been selected as the location server, the old one should retire to be a common node.

\subsection{Energy-aware GFG routing}

GHLS uses GFG routing to transmit data based on geographic location. The GFG routing is a greedy algorithm that selects the next-hop node whose location is currently closest to the destination in transmission range $R_{t}$. However, the node that is closest to the destination may have low residual energy, while the nodes around it still have sufficient energy. Based on this observation, the energy-aware GFG routing is proposed for load balance.

Consider a node $i$ that has to forward the data by the energy-aware GFG routing, the set of nodes that locate within the transmission range $R_{t}$ of node $i$ is defined as its neighbors $N(i)$. The distance from node $i$ to the destination $k$ is define as $d(i, k)$. Then we can define the set of its neighbor nodes whose location is closer to the destination as $N_{+}(i)$, and the average residual energy of $N_{+}(i)$ can be defined as $\bar{E}_{r+}(i)$

$$
\bar{E}_{r+}(i)=\frac{\sum_{j=1}^{M} E_{r}(j)}{M}, j \in N_{+}(i)
$$

Where $n_{+}(i)$ is the number of the nodes in $N_{+}(i)$ and $E_{r}(j)$ is the residual energy of the node $j$. Due to the broadcasting nature of wireless communication, each node can obtain $\bar{E}_{r+}$ by overhearing the data transmission. Then we define a variable named $E_{g}$.

$$
E_{g}(i)=g \times \bar{E}_{r+}(i)
$$

Where $g$ is a factor to control the impact of $\bar{E}_{r+}(i)$, and $g$ should be set between 0 and 1.

In the energy-aware GFG routing, the next-hop of node $i$ should be selected from $N_{+}(i)$ to ensure the convergence of the algorithm. Moreover, in order to balance the energy consumption, the residual energy of the next-hop node $j$ should be larger than $E_{g}$, i.e. $E_{r}(j)>E_{g}(i)$. At last, in the nodes that satisfy the conditions given above, the node with the minimum $d(i, k)$ is selected as the next-hop node. This condition en- 
sures the efficiency of the transmission path. To sum up, the selected next-hop node $n x$ should satisfy the condition that,

$$
d(n x, k)=\min \{d(i, k)\}, i \in N_{+}(i), E_{r}(i) \geq E_{g}
$$

The energy-aware GFG routing avoids selecting the low residual energy node as a relay in the routing path, and hence balances the energy consumption. How to determine the value of $g$ will be further discussed in Section 5.2.

\section{$5 \quad$ Simulation Results}

In this section, we evaluate the performance of GHLS- $\mathrm{R}^{2}$ via simulations. We use the network lifetime and residual energy ratio to evaluate the energy efficiency of the protocols. The network lifetime is defined as the time when the first node dies in the network. The residual energy ratio is defined as the ratio of residual energy to initial energy when the first node dies in the network.

The node density of the network is 0.01 node $/ \mathrm{m}^{2}$. The maximum transmission range $R_{t}$ is $60 \mathrm{~m}$. The velocity of the actuator node is $2 \mathrm{~m} / \mathrm{s}$. The update period is 30 seconds that is dividing the maximum transmission range by the actuator velocity to ensure network connectivity. The initial energy of every node is 50 units. The energy unit is defined as the energy consumed for broadcasting a unit packet in $R_{t}$. The events are generated with a fixed period $60 \mathrm{~s}$. The parameters setting for the simulation are summarized in Table 1.

Table 1. Simulation parameters

\begin{tabular}{|l|c|}
\hline \multicolumn{1}{|c|}{ Parameter } & Value \\
\hline Scaling factor of GHLS $(K)$ & 0 \\
\hline Initial energy of sensor nodes $\left(E_{S}\right)$ & 50 \\
\hline Side length of network area $(L)$ & $100-400$ \\
\hline Node density of network & $0.01 \mathrm{node}^{2} / \mathrm{m}^{2}$ \\
\hline Transmission range $\left(R_{t}\right)$ & $60 \mathrm{~m}$ \\
\hline Event generation period & $60 \mathrm{~s}$ \\
\hline Update period $\left(T_{u}\right)$ & $30 \mathrm{~s}$ \\
\hline Velocity of the actor $(v)$ & $2 \mathrm{~m} / \mathrm{s}$ \\
\hline
\end{tabular}

We firstly evaluate the impact of location server rotation and energy-aware GFG routing on the network lifetime and residual energy ratio, and then derive the guideline of setting the parameters in GHLS- $\mathrm{R}^{2}$. After that, we compare the performance of GHLS- $\mathrm{R}^{2}$ with that of GHLS to prove the efficiency of GHLS- $\mathrm{R}^{2}$. The simulations are implemented in Matlab, and each experiment is performed 50 times to obtain statistical results. 


\subsection{Impacts of rotation period}

In this section, we investigate the impact of rotation period. The rotation period is prolonged when $s$ in Eqn. 2 grows. The scaling factor $s$ varies from 1 to 20 . The network area varies from $200 * 200 \mathrm{~m}^{2}$ to $300 * 300 \mathrm{~m}^{2}$. The results are given in Fig. 3 and 4 .

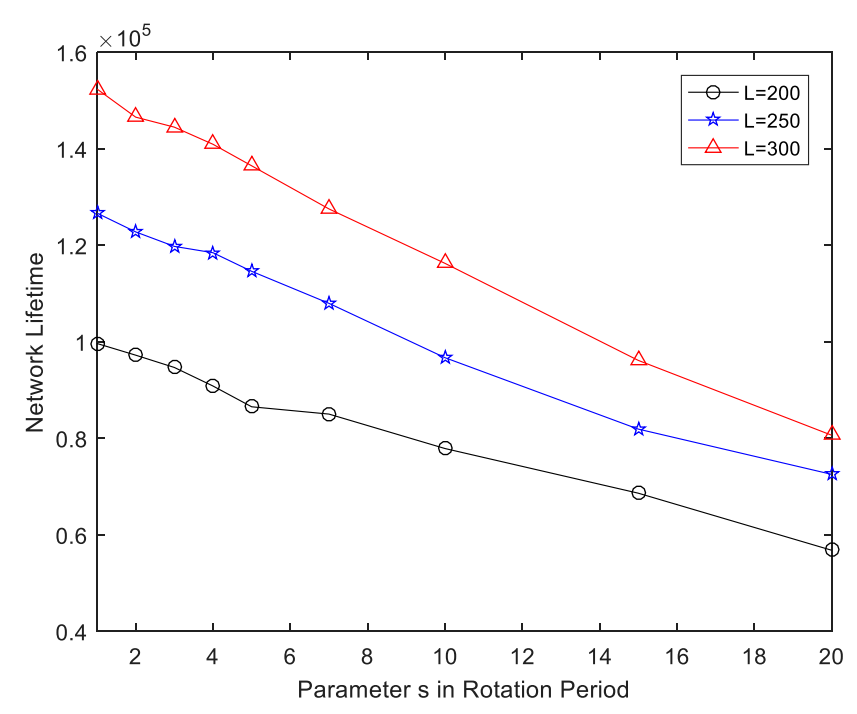

Fig. 3. The network lifetime with different rotation parameter $s$

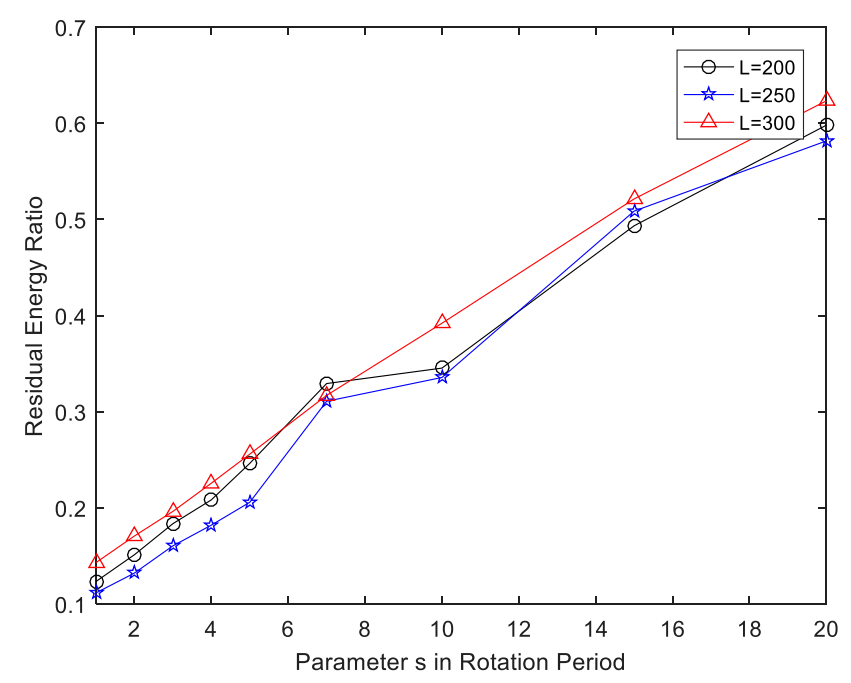

Fig. 4. The residual energy ratio with different rotation parameter $s$ 
Fig.3 and Fig.4 show that the rotation period has significant effects on network lifetime and residual energy ratio. As shown in Fig.3, the network lifetime decreases when the s grows. When $s=1$, the network lifetime is longest. When the network area is $300 * 300 \mathrm{~m}^{2}$ and $s=1$, the maximum network lifetime reaches up to $152408.4 \mathrm{~s}$. Moreover, as shown in Fig.4, when $s=1$, the residual energy ratio is lowest. The residual energy ratio is $14.32 \%$ when the network area is $300 * 300 \mathrm{~m}^{2}$ and $s=1$. The reason is that when the rotation period is prolonged, the selected nodes work as location server for a longer time. The location server consumes much more energy than other nodes in the rotation period. Hence, the energy consumption is more unbalanced when the rotation period grows.

As shown in the results, the network has longest lifetime and lowest residual energy ratio when $s=1$. Therefore, in the following simulations, the $s$ is determined as 1 .

\subsection{Impacts of energy-aware GFG routing}

In this section, the simulations are used to evaluate the impact of the energy-aware GFG routing. In the energy aware GFG routing, the value of $E_{g}$ in Eqn.4 is determined by the scaling factor $g$. It represents the importance of residual energy in the routing decision, and it should be no larger than 1 to guarantee that the next-hop node can be found. In the simulations, the scaling factor $g$ varies from 0 to 1 , and the network area grows from $200 * 200 \mathrm{~m}^{2}$ to $300 * 300 \mathrm{~m}^{2}$. Fig. 5 and 6 give the simulation results.

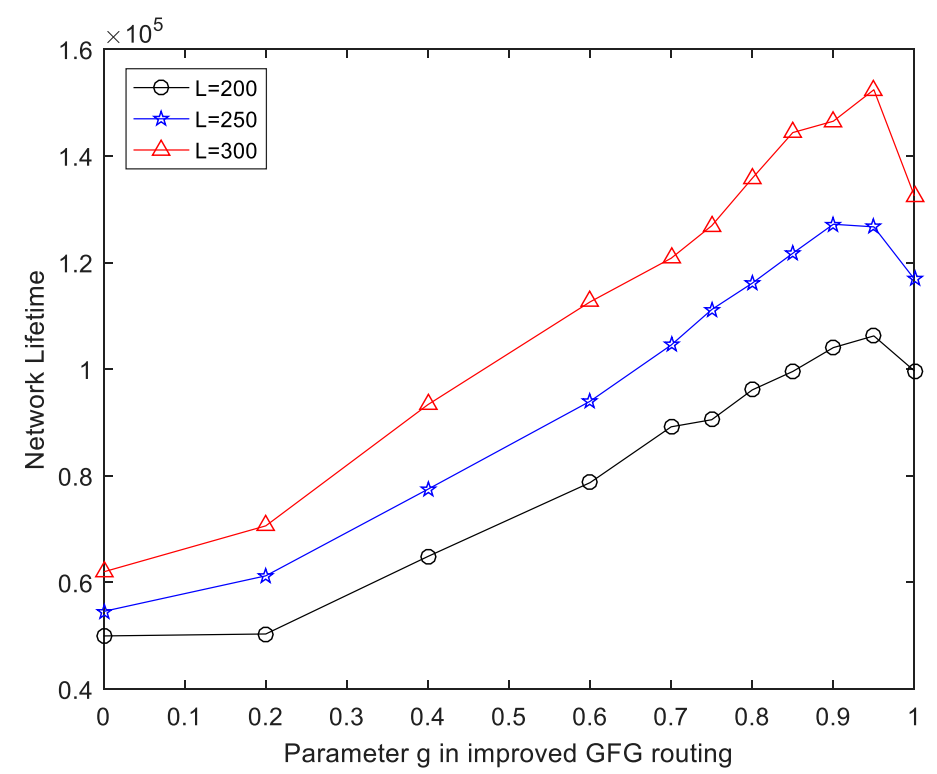

Fig. 5. The lifetime with different routing parameter $g$ 


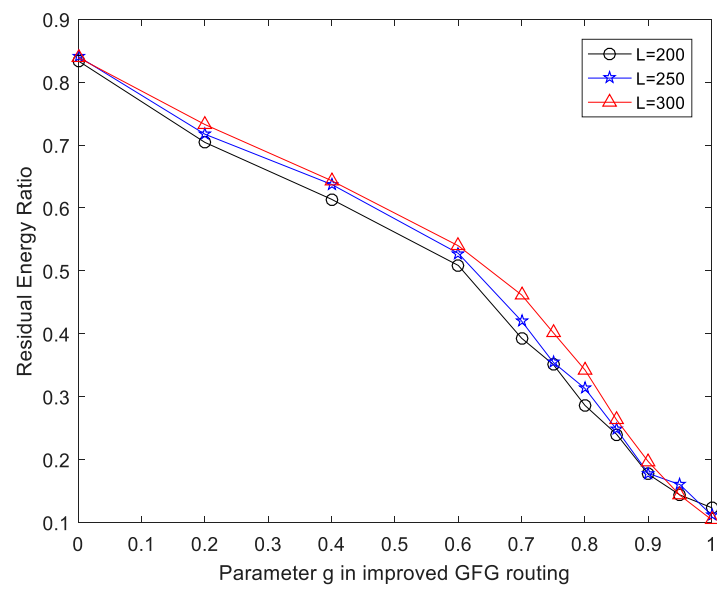

Fig. 6. The residual energy ratio with different routing parameter $g$

As shown in Fig.5, the network lifetime reaches maximum when $g=0.95$. On the other hand, Fig. 6 shows that the residual energy ratio is lowest when $g=1$. These results show that increasing the factor $g$ is helpful to balance the energy consumption. Nevertheless, the cost is the energy efficiency of the transmission path. When $g>$ 0.95 , the cost exceeds the benefits, which results in the reduction of network lifetime.

Moreover, we also execute simulations to study the joint effect of the rotation parameter $s$ and the routing parameter $g$. The side length of network area is fixed at $L=$ 300 . The rotation parameter $s$ grows from 1 to 5 , and the routing parameter $g$ varies from 0.7 to 1 . The results are given in Fig.7 and 8. It is clear to see that the network lifetime reaches maximum when $s=1$ and $g=0.95$. This result matches the results given in Fig. 3 and5. Therefore, we set $s=1$ and $g=0.95$ in the following simulations.

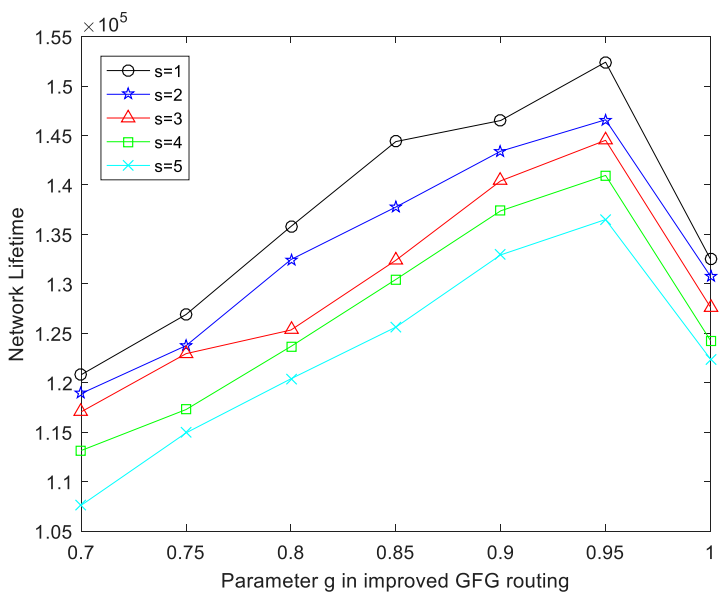

Fig. 7. The residual energy ratio with different $g$ and $s$ 


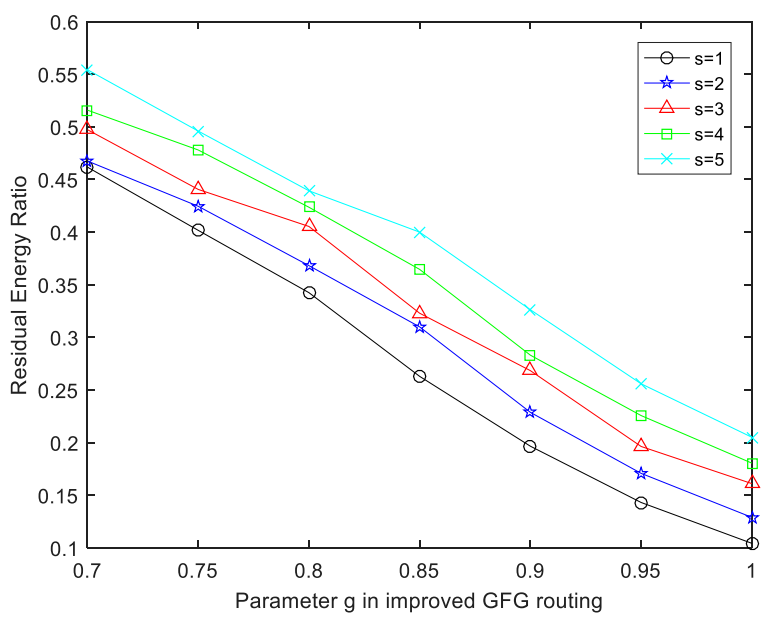

Fig. 8. The residual energy ratio with different $g$ and $s$

\subsection{Network lifetime comparison}

In this section, the performance of GHLS- ${ }^{2}$ is compared with that of GHLS to prove its efficiency. In order to evaluate the effect of location server rotation and energy-aware GFG routing respectively, we implement the GHLS-R that only adopts the location server rotation, and the GHLS- $\mathrm{R}^{2}$ that adopts both location server rotation and energy-aware GFG routing. The simulations are executed to compare the network lifetime and residual energy ratio of GHLS, GHLS-R and GHLS- ${ }^{2}$. The simulation results are given in Fig.9 and Fig.10.

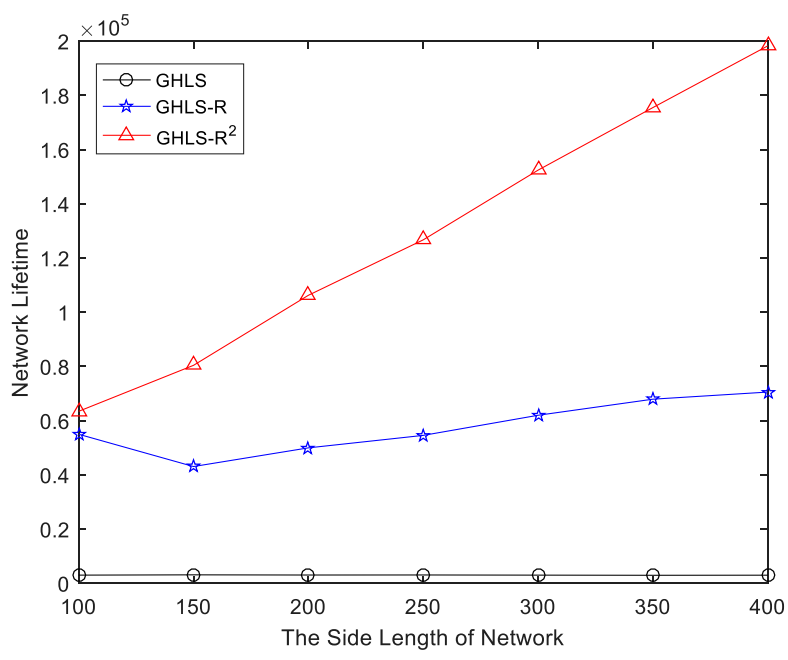

Fig. 9. The lifetime with different network area 


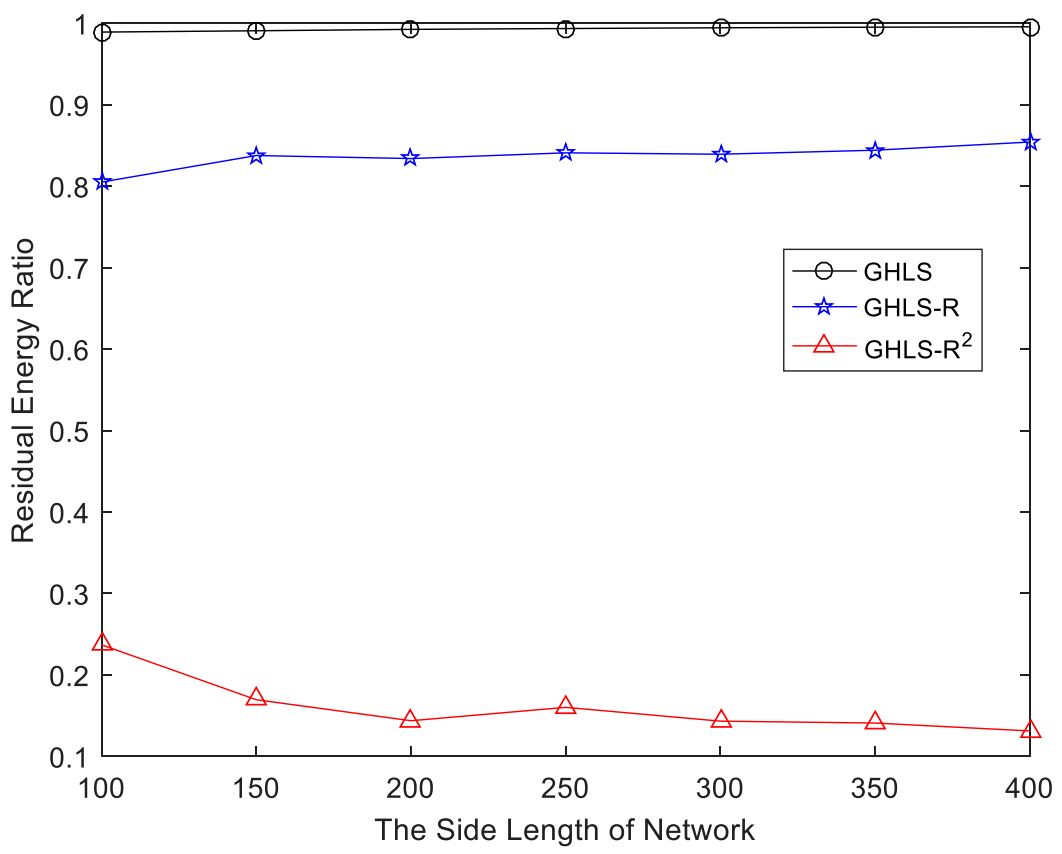

Fig. 10.The residual energy ratio with different network area

As shown in Fig.9 and Fig.10, GHLS has the lowest network lifetime which is about 3000 s, and the residual energy ratio of GHLS are greater than $98 \%$. According to the statistics of dead nodes, $100 \%$ first dead nodes are location servers which suffers from heavy traffic load. Thus, the energy consumption is severe unbalanced in GHLS.

With the help of location server rotation, GHLS-R increases the network lifetime from 18 to 23 times by comparing with GHLS. It is because, in GHLS-R, the location server rotates and more nodes become location server in turns to balance the energy consumption. However, the residual energy ratio is $80 \%-86 \%$ which means a great amount of energy is wasted when the first dead nodes appears. According to the statistics of dead nodes, less than $10 \%$ dead nodes are location servers in GHLS-R.

In GHLS- ${ }^{2}$ which adds energy-aware GFG routing based on GHLS-R, the network lifetime is 1.2 to 3 times longer than that of GHLS-R. Moreover, the residual energy ratio reduces to a reasonable level at $13 \%-24 \%$. Compared with GHLS-R, this result proves the importance of energy-aware GFG routing for balancing the energy consumption. Moreover, as shown in Fig.9 and Fig.10, the advantages of GHLS$\mathrm{R}^{2}$ are bigger in a larger network area which proves that GHLS- $\mathrm{R}^{2}$ improves the scalability in WSAN. 


\section{Conclusion}

In this paper, we study the unbalanced energy consumption of GHLS in WSAN, and then propose an improved protocol named GHLS- $\mathrm{R}^{2}$ to achieve load balance. In GHLS-R ${ }^{2}$, the location server rotation and the energy-aware GFG routing effectively balance the energy consumption and hence prolong the network lifetime. The simulation results prove the efficiency of GHLS- $\mathrm{R}^{2}$.

\section{$7 \quad$ Acknowledgement}

This work is partially supported by NSF of China (61673116, 61304260, 61703106).

\section{$8 \quad$ References}

[1] I. F. Akyildiz and I. H. Kasimoglu, "Wireless sensor and actor networks: research challenges," Ad Hoc Networks, vol. 2, no. 4, pp. 351 -367, 2004 https://doi.org/10.1016/j.adho c.2004.04.003

[2] S. Zhu, C. Chen, X. Ma, B. Yang, and X. Guan, "Consensus based estimation over relay assisted sensor networks for situation monitoring." IEEE Journal of Selected Topics in Signal Processing, vol. 9, no. 2, pp.278-291, 2015. https://doi.org/10.1109/JSTSP.2014.23 $\underline{75851}$

[3] Z. Xu, L. Chen, C. Chen, and X. Guan, "Joint Clustering and Routing Design for Reliable and Efficient Data Collection in Large-Scale Wireless Sensor Networks," IEEE Internet Things Journal, vol. 3, no. 4, pp. 520-532, 2016 https://doi.org/10.1109/JIOT.2015.24823 $\underline{63}$

[4] S. M. Das, H. Pucha, and Y. C. Hu, "On the scalability of rendezvous-based location services for geographic wireless ad hoc routing," Computer Networks, vol. 51, no. 13, pp. 3693-3714, 2007 https://doi.org/10.1016/j.comnet.2007.03.006

[5] J. Li, J. Jannotti, D. S. J. D. Couto, D. R. Karger, and R. Morris, "A scalable location service for geographic ad hoc routing," in International Conference on Mobile Computing and Networking, 2000, pp. 120-130 https://doi.org/10.1145/345910.345931.

[6] M. Luki and I. Mezei, "Localised querying and location update service in wireless sensor and robot networks with arbitrary topology," International Journal of Ad Hoc \& Ubiquitous Computing, vol. 22, no. 1, pp. 48-61, 2016. https://doi.org/10.1504/IJAHUC.2016.07 $\underline{6593}$

[7] P. Bose, P. Morin, and J. Urrutia, "Routing with guaranteed delivery in ad hoc wireless networks," Wireless Networks, vol. 7, no. 6, pp.609-616, 2001. https://doi.org/10.1504/IJ AHUC.2016.076593

[8] D. Liu, I. Stojmenovic, and X. Jia, "A scalable quorum based location service in ad hoc and sensor networks," in IEEE International Conference on Mobile Adhoc and Sensor Systems, 2008, pp. 489-492. https://doi.org/10.1504/IJCNDS.2008.017205

[9] Li, Jinyang, Jannotti, John, D. Couto, D. S. J, Karger, D. R, Morris, and Robert, “A scalable location service for geographic ad hoc routing," Mobicom Boston Ma, pp. 120-130, 2008. 
[10] X. Tian, W. Li, Y. Yang, Z. Zhang, and X. Wang, "Optimization of Fingerprints Reporting Strategy for WLAN Indoor Localization,” IEEE Transactions on Mobile Computing, vol. 17, no. 2, pp. 390-403, 2018. https://doi.org/10.1109/TMC.2017.2715820

[11] Z. Xu, G. Liu, H. Yan, B. Cheng, and F. Lin, "Trail-Based Search for Efficient Event Report to Mobile Actors in Wireless Sensor and Actor Networks," Sensors, vol. 17, no. 12, p. 2468, 2017. https://doi.org/10.4108/tridentcom.2008.3155

[12] Z. Xu, C. Chen, B. Cheng, and X. Guan, "Sharing mobility strategy improves location service in wireless sensor and actor networks," IEEE Communications Letters, vol. 16, no. 6, pp. 858-861, 2012. https://doi.org/10.1109/LCOMM.2012.041112.120346

[13] G. Liu, Z. Xu, H. Yan, H. Jiang, C. Chen, and L. Yu, "Trail-based location service in event-driven wireless sensor and actor networks," in 2017 IEEE 86th Vehicular Technology Conference (VTC-2017Fall), Sept 2017, pp. 1-5. https://doi.org/10.11 09/VTCFall.2017.8288181

[14] G. Wang, T. Wang, W. Jia, and M. Guo, "Local update-based routing protocol in wireless sensor networks with mobile sinks," in IEEE International Conference on Communications, 2007, pp. 3094-3099. https://doi.org/10.1109/ICC.2007.514

[15] G. Wang, T. Wang, W. Jia, M. Guo, and J. Li, "Adaptive location updates for mobile sinks in wireless sensor networks," Journal of Supercomputing, vol. 47, no. 2, pp. 127145, 2009. https://doi.org/10.1007/s11227-008-0181-5

[16] J. Lee, J. Y. Jang, E. Lee, and S. H. Kim, "Efficient sink location service for prolonging the network lifetime in wireless sensor networks," in IEEE Consumer Communications and Networking Conference, 2016, pp. 290-291https://doi.org/10.110 9/CCNC.2016.7444785

[17] R. Khadim, M. Erritali, and A. Maaden, "Hierarchical location-based services for wireless sensor networks," in International Conference on Computer Graphics, Imaging and Visualization, 2016, pp. 457-463. https://doi.org/10.1109/CGiV.2016.97

[18] E. B. Hamida and G. Chelius, "A line-based data dissemination protocol for wireless sensor networks with mobile sink," in IEEE International Conference on Communications, 2009, pp. 2201-2205. https://doi.org/10.1109/ICC.2008.420

[19] Y. Zeng, K. Xiang, D. Li, and A. V. Vasilakos, "Directional routing and scheduling for green vehicular delay tolerant networks," Wireless Networks, vol. 19, no. 2, pp. 161-173, 2013. https://doi.org/10.1007/s11276-012-0457-9

[20] T. Camp, J. Boleng, and V. Davies, "A survey of mobility models for ad hoc network research,” Wireless Communications \& Mobile Computing, vol. 2, no. 5, pp. 483-502, 2002. https://doi.org/10.1002/wcm.72

[21] N. Zhou, R. Chen, Y. Xia, J. Huang, G. Wen, "Neural network-based reconfiguration control for spacecraft formation in obstacle environments," International Journal of Robust and Nonlinear Control, vol. 28, no. 6, pp. 2442-2456, 2018. https://doi.org/10.1002/rnc.40 $\underline{25}$

[22] M. D. Francesco, G. Anastasi, M. Conti, S. K. Das, and V. Neri, "Reliability and energyefficiency in ieee 802.15.4/zigbee sensor networks: An adaptive and cross-layer approach," IEEE Journal on Selected Areas in Communications, vol. 29, no. 8, pp. 1508-1524, 2011. https://doi.org/10.1109/JSAC.2011.110902

[23] P. Santi, "The critical transmitting range for connectivity in mobile ad hoc networks," IEEE Transactions on Mobile Computing, vol. 4,no. 3, pp. 310-317, 2005. https://doi.org/10.1109/TMC.2005.45

[24] S. He, J. Chen, Y. Sun, "Coverage and Connectivity in Duty-Cycled Wireless Sensor Networks for Event Monitoring,” IEEE Trans. Parallel Distrib. Syst. vol.23,no 3, pp. 475-482, 2012. https://doi.org/10.1109/TPDS.2011.191 


\section{Authors}

Zhaolin Wang is an undergraduate student with the College of Electrical and Automation in Fuzhou University, Fuzhou, China. His research interests include Wireless Sensor and Actor Networks.

Zhezhuang Xu received the Ph.D degree in Control and Systems from Shanghai Jiao Tong University, Shanghai, China in 2012. He is currently an Associate Professor with the School of Electrical Engineering and Automation, Fuzhou University, Fuzhou, China. His research interests include Wireless Sensor and Actuator Network, and their applications in Industrial Internet of Things. He has authored and/or coauthored over 30 referred international journal and conference papers.

Renxu Xie received the B.E. degree from Fuzhou University. He is currently a postgraduate student with the College of Electrical Engineering and Automation in Fuzhou University, Fuzhou, China. His research interests include Industrial big data and Internet of things.

Haotian Yan received the B.E. degree from Fuzhou University. He is currently a postgraduate student with the Department of Electronic Information and Engineering in Hong Kong Polytechnic University, Hong Kong, China. His research interests include Wireless Sensor and Actor Networks.

Article submitted 2018-08-24. Resubmitted 2018-11-07. Final acceptance 2018-11-12. Final version published as submitted by the authors. 